Supporting Information for:

\title{
Selection of color-changing and intensity-increasing fluorogenic probe as protein-specific indicator obtained via the 10BASE ${ }_{d}-T$
}

Masumi Taki ${ }^{*}{ }^{\dagger}$, Hiroaki Inoue ${ }^{\dagger}$, Kazuto Mochizuki ${ }^{\dagger}$, Jay Yang ${ }^{\S}$, and Yuji Ito

${ }^{\dagger}$ Department of Engineering Science, Bioscience and Technology Program, The Graduate School of Informatics and Engineering, The University of Electro-Communications (UEC), 1-5-1 Chofugaoka, Chofu, Tokyo 182-8585, Japan $\S$ Department of Anesthesiology, University of Wisconsin, School of Medicine and Public Health, Madison, WI 53706 USA

* Department of Chemistry and Bioscience, Graduate School of Science and Engineering, Kagoshima University, 1-21-35 Korimoto, Kagoshima, Kagoshima 890-0065, Japan

$$
\text { E-mail: taki@pc.uec.ac.jp (M.T.) }
$$

Fax: +81-42-443-5501. Phone: +81-42-443-5980.

Supplemental figures 


\section{Supplemental figures}

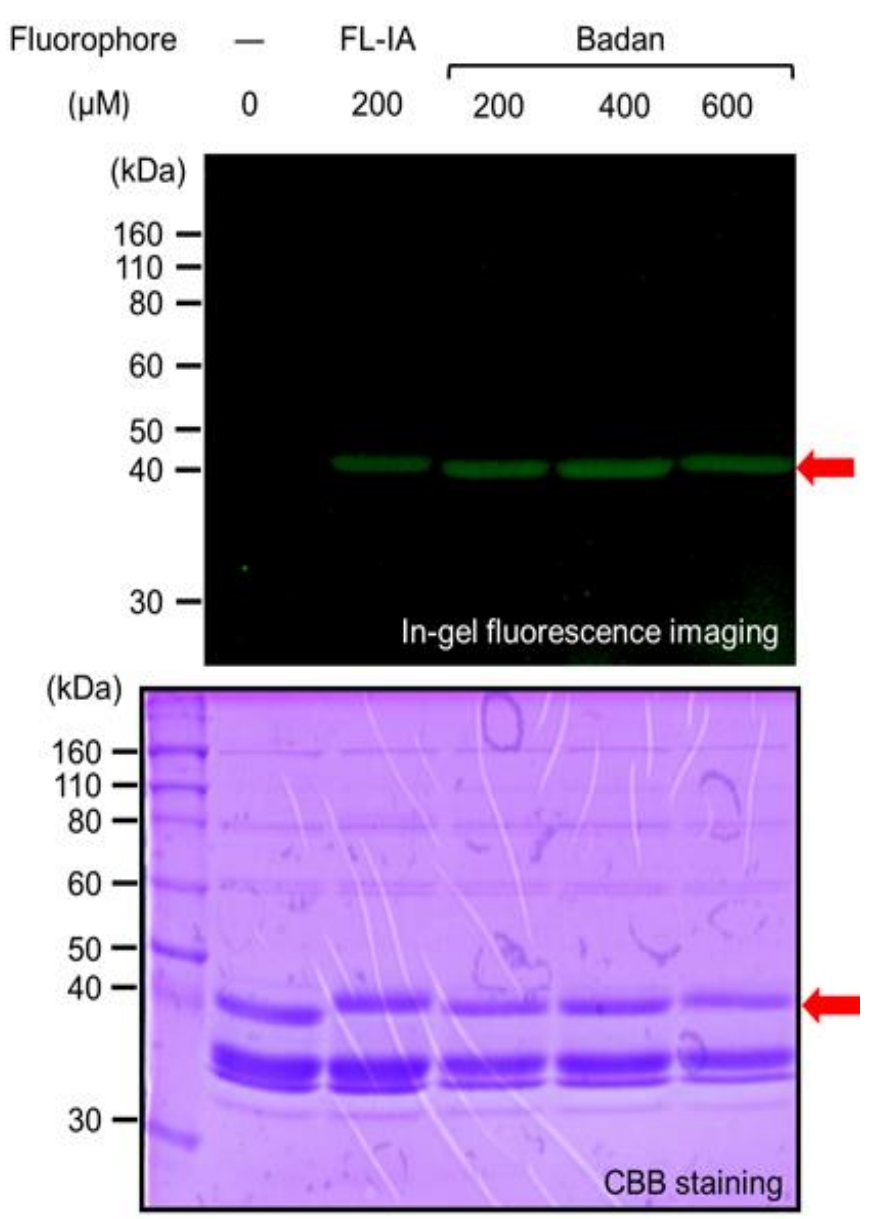

Figure S1. Confirmation of appropriate $10 \mathrm{BASE}_{\mathrm{d}}-\mathrm{T}$ on a $\mathrm{T} 7$ phage-displayed model peptide and optimization of Badan concentration. A model peptide with the sequence GSRVSCGGRDRPGCLSV displayed on phage was modified via the $10 \mathrm{BASE}_{\mathrm{d}}-\mathrm{T}$ with various molar concentrations of Badan or Fluorescein-iodoacetamide (FL-IA; as a positive control for the $10 \mathrm{BASE}_{\mathrm{d}}-\mathrm{T}$ ) under standard conditions. Equal amounts of phage proteins were subjected to sodium dodecyl sulfate-polyacrylamide-gel electrophoresis (SDS-PAGE) followed by fluorescence imaging. The arrow indicates fluorescently-labeled peptide displayed on gp10 capsid protein of phage. (A) In-gel fluorescence imaging after the gel electrophoresis. From the result, optimum Badan concentration was determined to be above $0.2 \mathrm{mM}$. (B) Total T7 phage proteins were stained with Coomassie brilliant blue (CBB). 


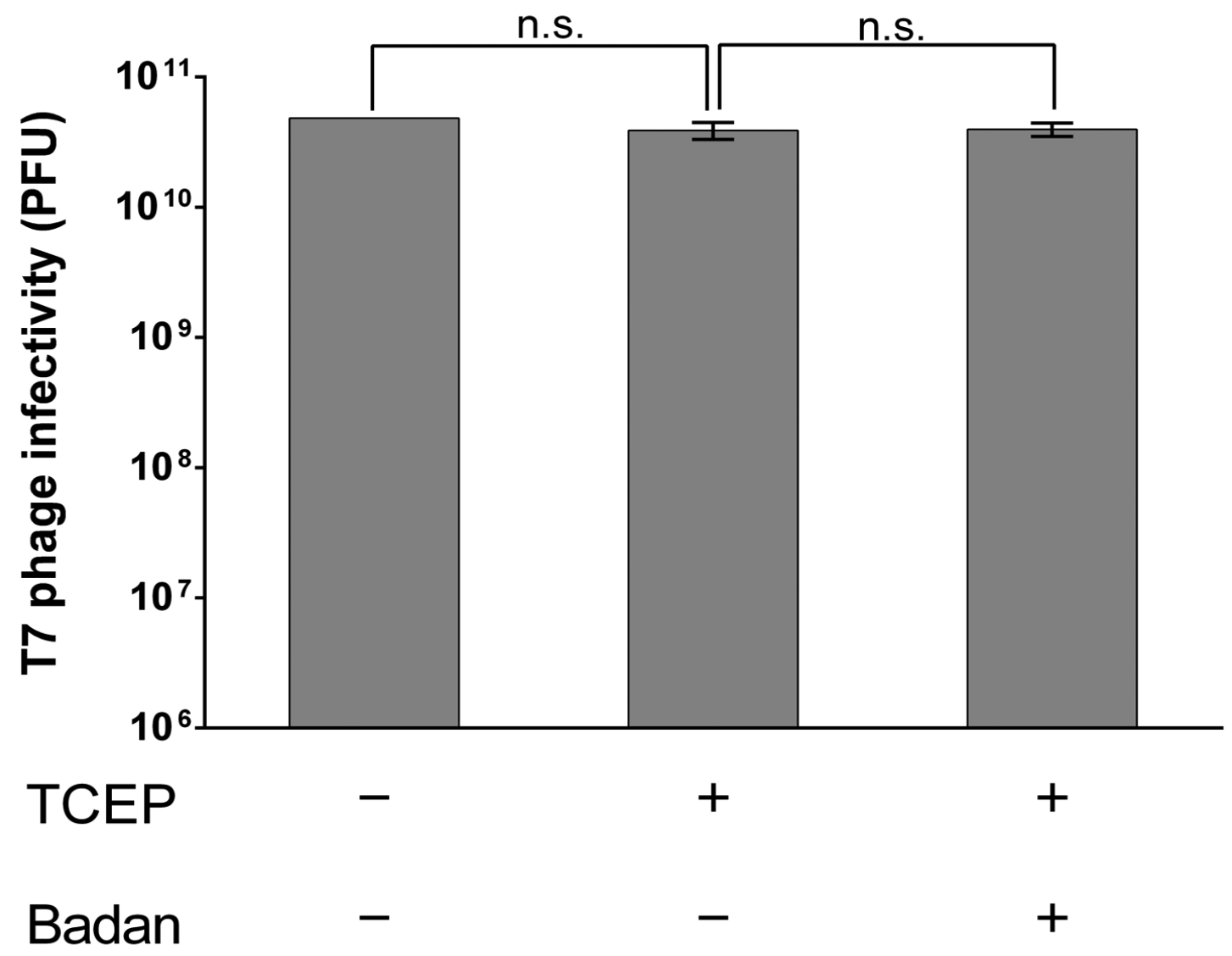

Figure S2. Infectivity of modified $\mathrm{T} 7$ phage. A $\mathrm{T} 7$ phage library (-S-G-G-G-X $\mathrm{X}_{3}-\mathrm{C}-\mathrm{X}_{5-7}-\mathrm{C}-\mathrm{X}_{3} ; \mathrm{X}$ represents any randomized amino acid) was treated with or without Badan in the presence of tris(2-carboxyethyl)phosphine (TCEP) under standard condition of the $10 \mathrm{BASE}_{\mathrm{d}}-\mathrm{T}$. The number of plaque forming units was determined by a serial dilution method and plaque assay. The graph summarizes the results of three independent experiments. Error bars represent standard deviations. Statistical analysis was performed by unpaired Student's $t$-test. n.s., not significant. 
2 - A

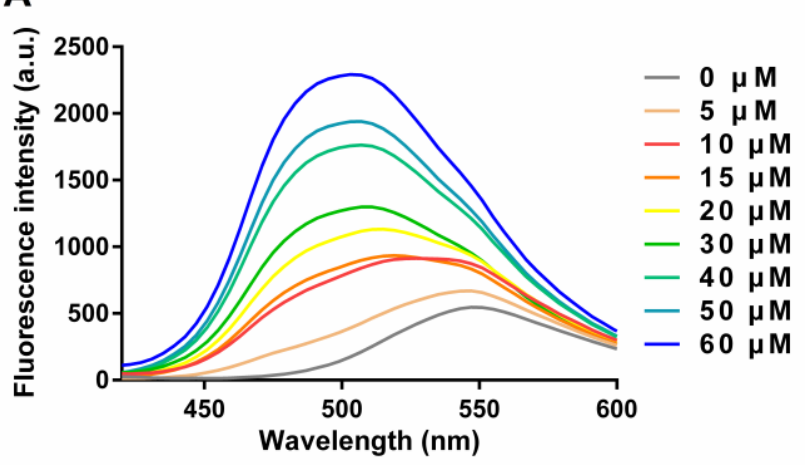

$\underline{3}-\mathbf{A}$

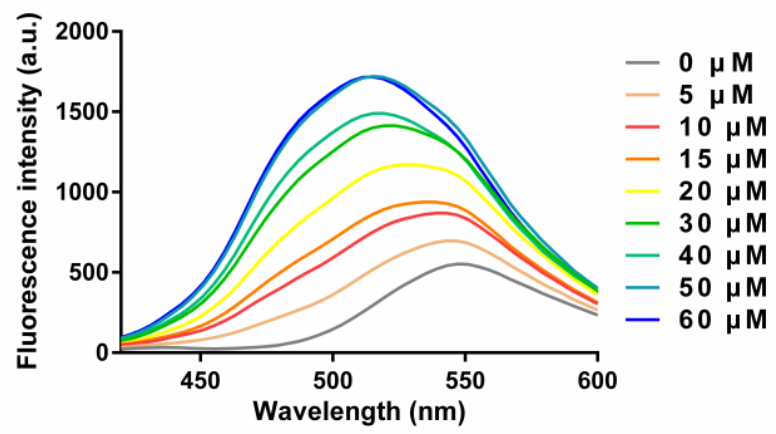

$\underline{2}$ - B

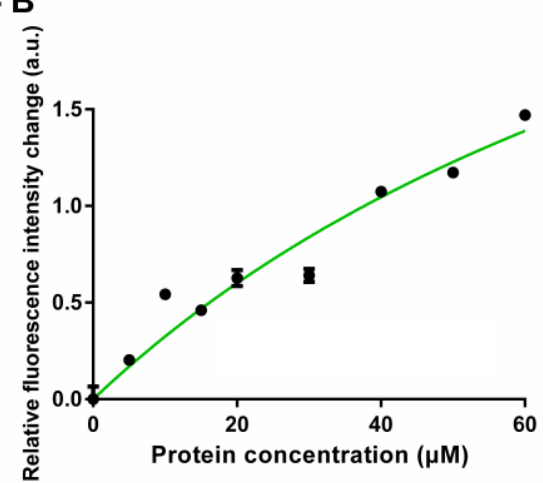

$\underline{3}$ - B

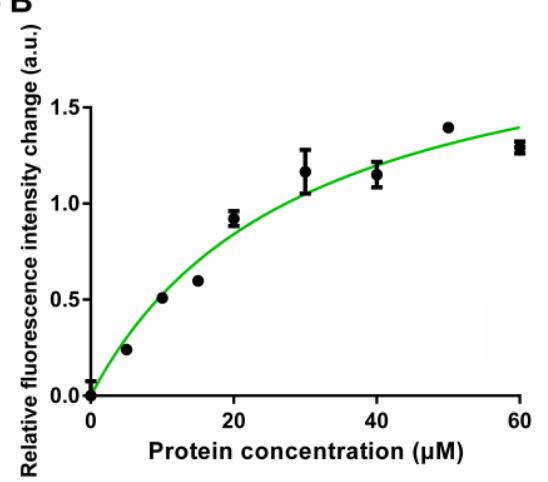

Figure S3. (A) Fluorescence titration upon GST-binding and (B) relative fluorescence intensity change at $551 \mathrm{~nm}$ for fluorogenic probes $\underline{\mathbf{2}}$ and $\underline{\mathbf{3}}$. The amino acid sequences of $\underline{\mathbf{2}}$ and $\underline{\mathbf{3}}$ are $\mathrm{NNPC} * \mathrm{TGF}$ and $\mathrm{PGPC} * \mathrm{G}$, respectively. $\mathrm{C} *$ represents Prodan-conjugated cysteine. The spectrum corresponding to NTVSC*HGF (1) is shown in Fig. 2 in the main text. When an actual detection limit is defined to be a hypsochromic shift over 25 $\mathrm{nm}$ of the maximum emission wavelength, those for $\underline{\mathbf{1}}$ and $\underline{\mathbf{2}}$ / $\underline{\mathbf{3}}$ are 2 and $20 \mu \mathrm{M}$ (i.e. 4 and $40 \mathrm{pmol}$ ) using NanoDrop 3300 fluorospectrometer. 

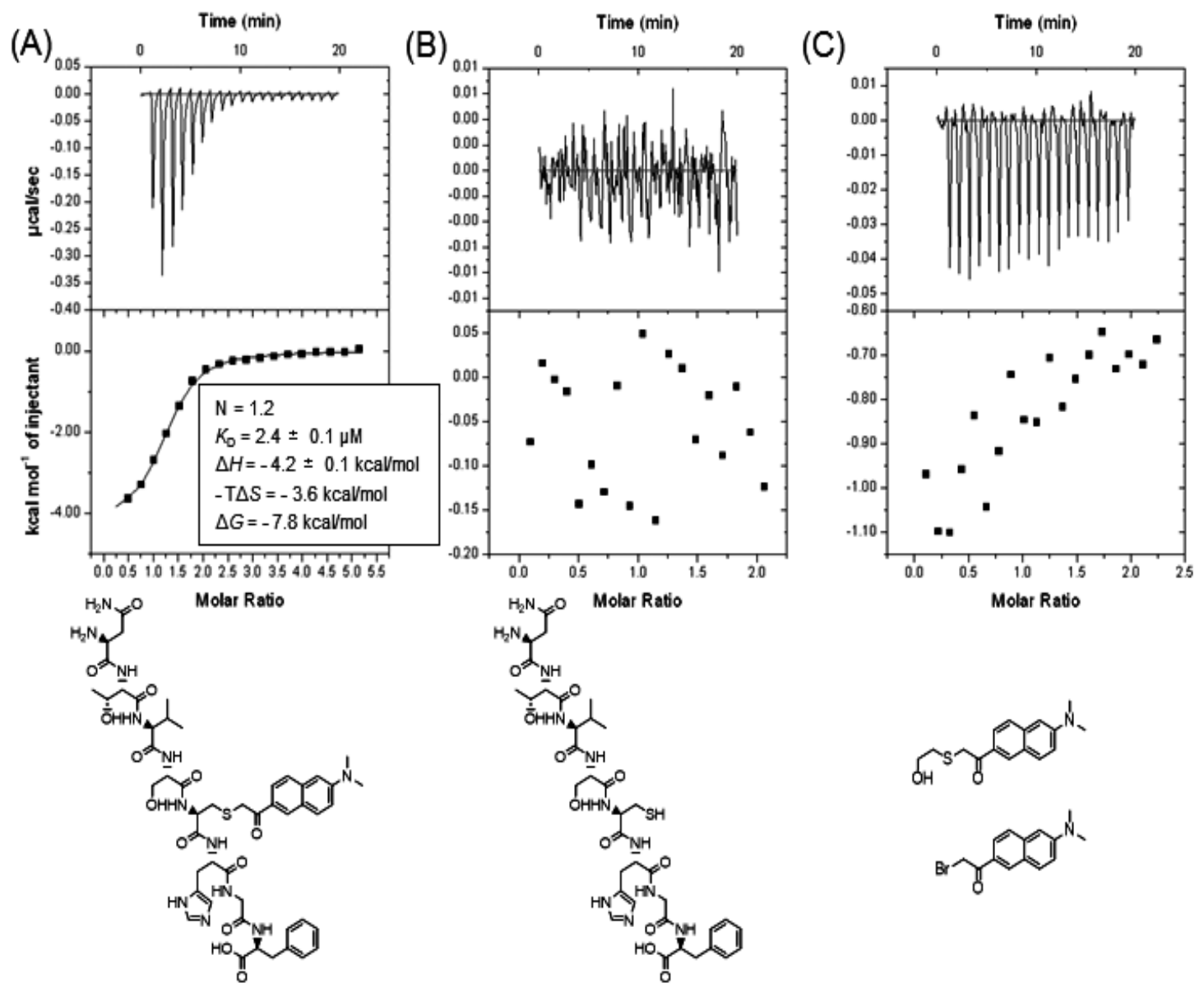

Figure S4. Isothermal titration calorimetry (ITC) profiles of titrations of GST with (A) Prodan-evolver $\underline{\mathbf{1}}$, (B) linear surrounding peptide with the same peptide sequence without Prodan conjugation, and (C) 1:1 (mol:mol) mixture of Badan and its 2-mercaptoethanol adduct. $\mathrm{N}$ : number of binding sites, $K_{\mathrm{D}}$ : dissociation constant, $\Delta \mathrm{H}$ : enthalpy change, $\Delta \mathrm{S}$ : entropy change, $\Delta \mathrm{G}$ : free energy change. 


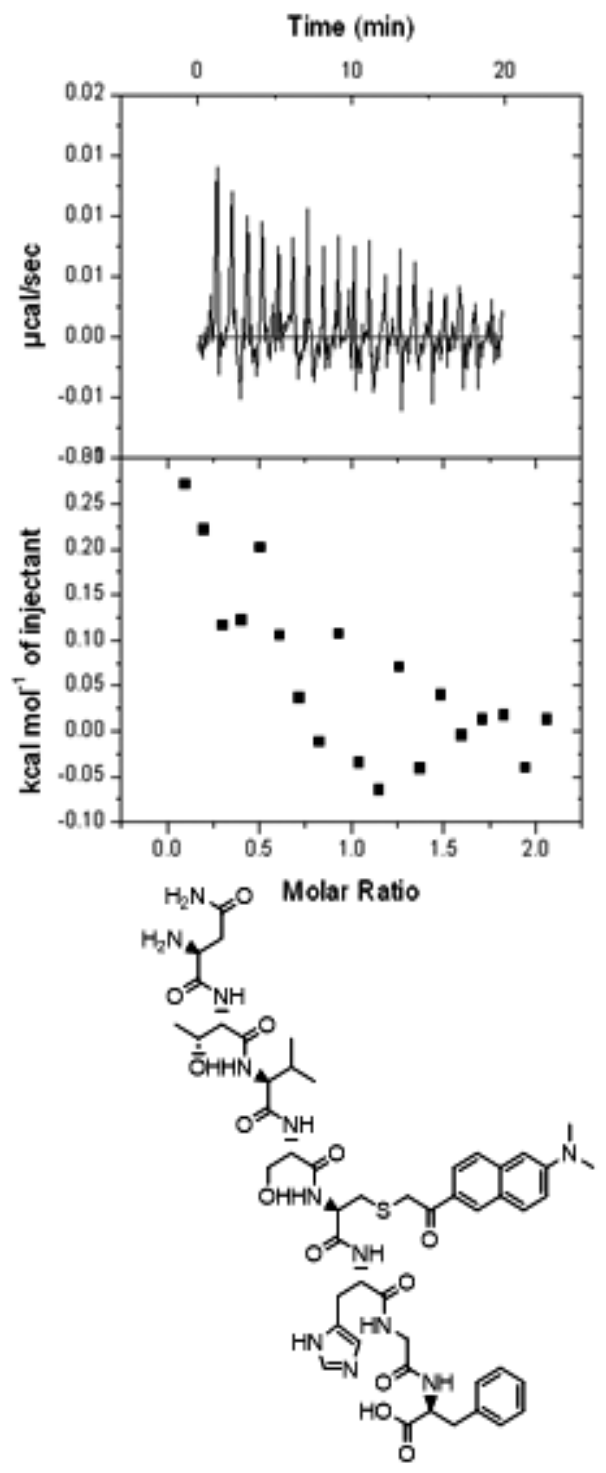

Figure S5. Isothermal titration calorimetry (ITC) profiles of titrations of streptavidin (target unrelated protein) with Prodan-evolver $\underline{1}$. 

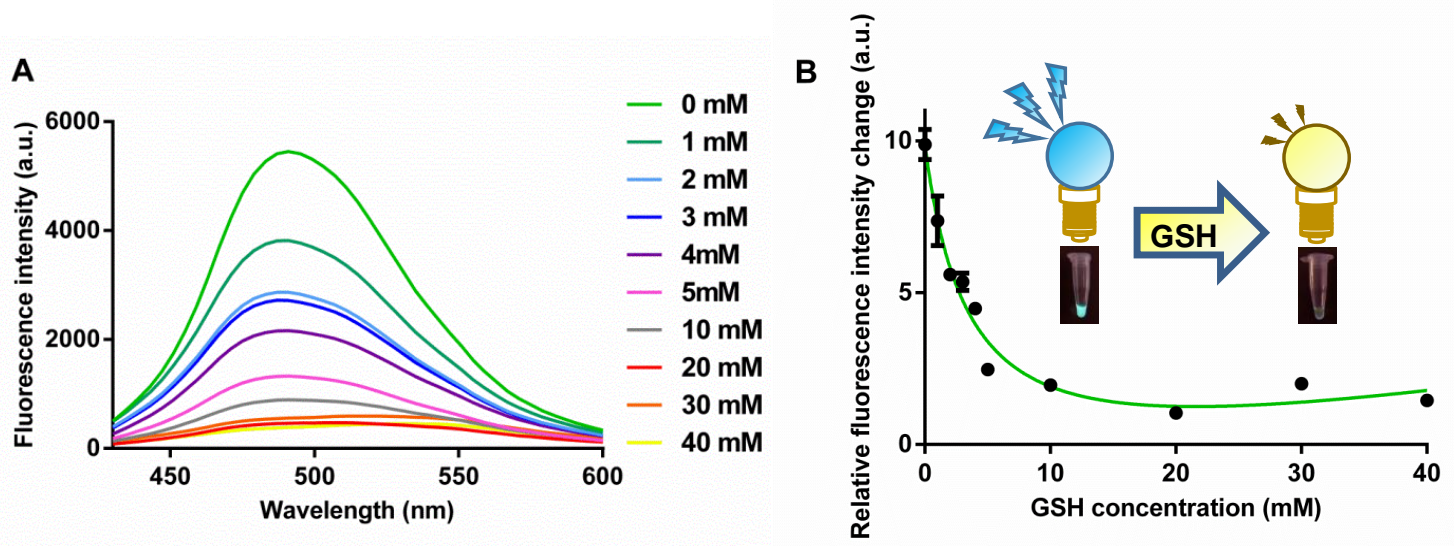

Figure S6. Competition assay measured by fluorescence spectroscopy. (A) Fluorescence titration and (B) relative fluorescence intensity change at $551 \mathrm{~nm}$. Glutathione (GSH) is a GST-binder, and the interaction between $\underline{\mathbf{1}}$ and GST was disrupted in the presence of GSH in a concentration dependent manner. The bright cyan fluorescence turned back to a weak yellow signal. 


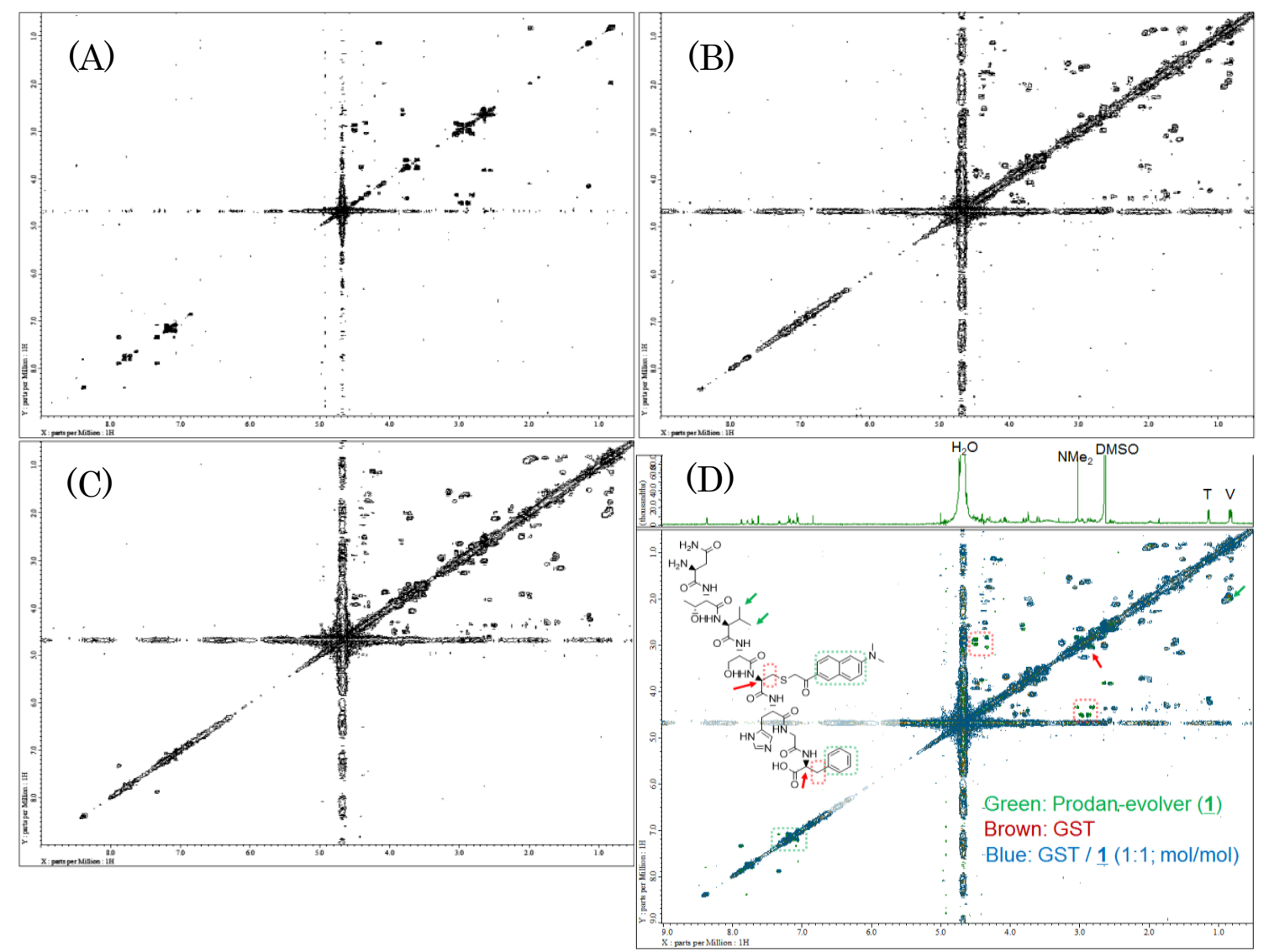

Figure S7. ${ }^{1} \mathrm{H}-{ }^{1} \mathrm{H}$ correlation spectroscopy (COSY) spectra of (A) Prodan-evolver $\underline{\mathbf{1}}$ alone $(0.14 \mathrm{mM}),(B)$ GST alone $(0.14 \mathrm{mM}),(\mathrm{C})$ both of them $(0.14 \mathrm{mM}$ each). (D) Their superimposed spectrum is summarized with a 1D proton nucleic magnetic resonance (NMR) spectrum of $\underline{\mathbf{1}}$ alone. The chemical structure of $\underline{\mathbf{1}}$ is also shown in the figure. Upon binding, aromatic- and $\alpha / \beta$-carbon-attached proton signals (shown in green square dots, red arrows, and red square dots, respectively) were broadened, and methyl protons of valine (green arrows) were slightly shifted. 


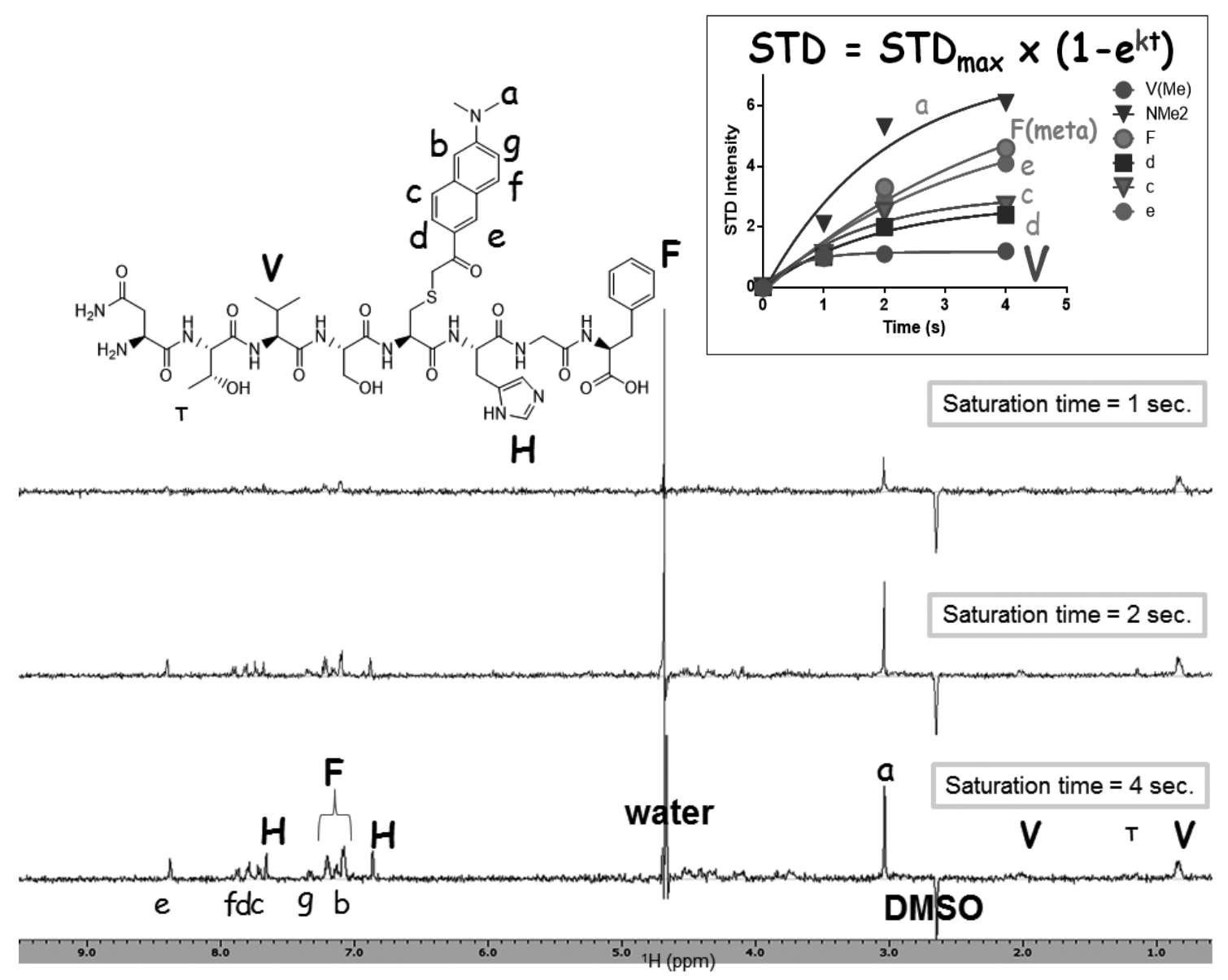

Figure S8. Quantitative epitope mapping of the Prodan-evolver (1) upon GST binding on the basis of saturation transfer difference-nucleic magnetic resonance (STD-NMR) measurement. Saturation time was varied in each measurement, and the relative STD signal intensities (STD) of these protons-of-interest were plotted against the saturation time. Exponential curve fitting of the plot was shown in the inset; relative saturation rate constant of each proton $(\mathrm{k})$ was obtained from STD at each saturation time $(\mathrm{t})$ and maximum STD signal intensity $\left(\mathrm{STD}_{\max }\right)$. 


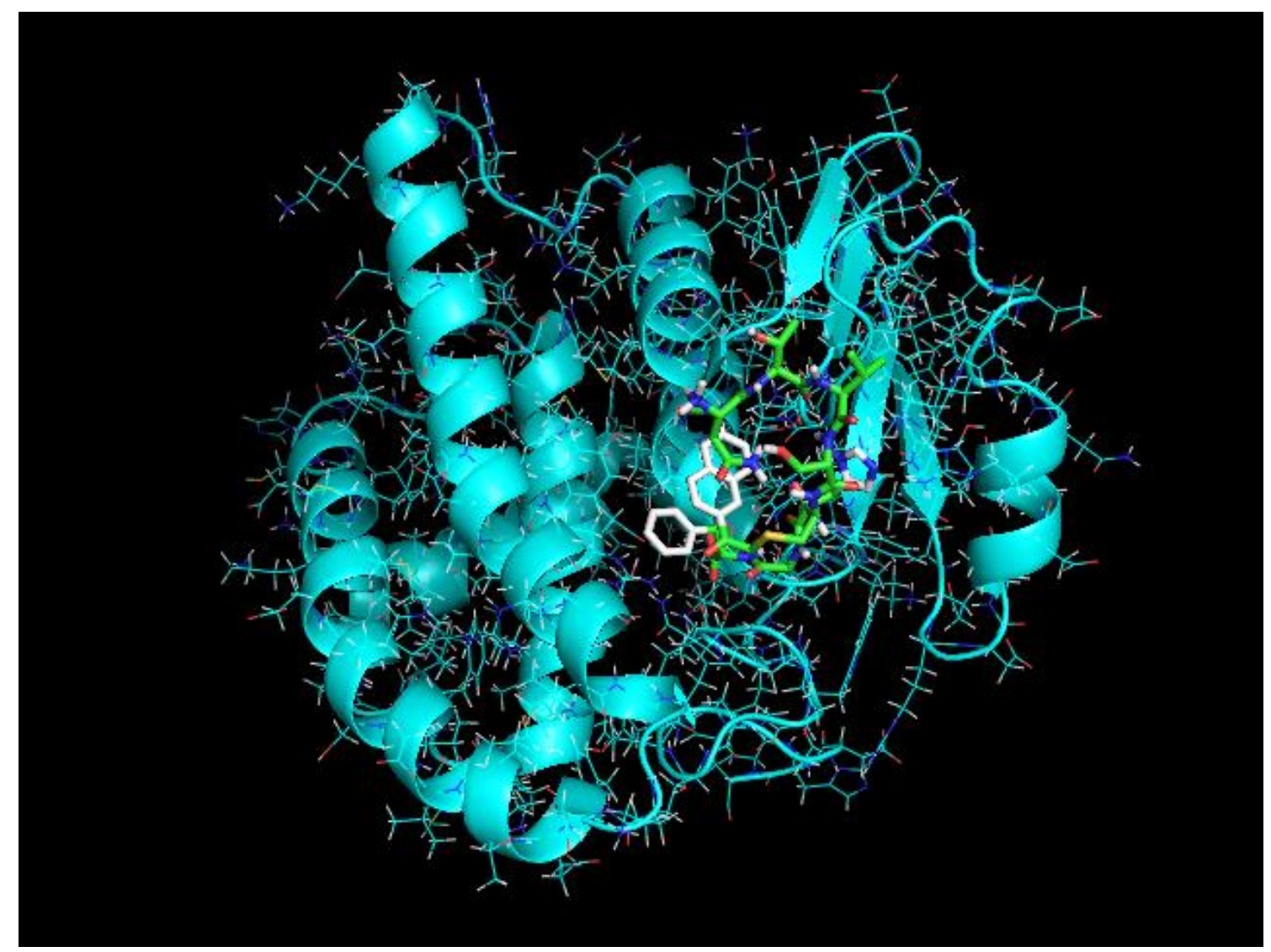

Figure S9. Best docking model between GST (PDB ID: 1UA5) and Prodan-evolver (1) with a lowest binding energy of $-7.1 \mathrm{kcal} / \mathrm{mol}$ comparable to the natural ligand GSH. Note that all of the important hydrophobic moieties found by STD-NMR measurements (Fig. 3 in the body text) were in close proximity of atoms of the GSH-binding pocket. The Prodan moiety rested in between the polypeptide of the ligand and the GSH-binding pocket of GST, while valine showed close proximity to residues N53, F45 and the phenylalanine to residues Y103, S106 of GST, respectively, perhaps contributing to the observed broadening of the NMR signal in Fig. S7. 


\section{Materials and methods}

\section{General}

All experiments were performed with commercially available reagents and kits. A commercially-available synthon (6-Bromoacetyl-2-dimethylaminonaphthalene; Badan) was purchased from Toronto Research Chemicals Inc (Toronto, ON, Canada). The derived fluorogenic core structure after its thioetherification, namely Prodan, possesses the 6-acetyl-2-dimethylaminonaphthalene unit, and it does not have 6-propionyl-2-dimethylaminonaphthalene in the strict sense; we chose to use the former common name because of its popularity within broad disciplines. Remarkable solvatochromic effect of Prodan by changing solvent polarity has been already compared to other environmentally-sensitive fluorophores and well summarized in a recent review. According to the paper, fluorescence maximum wavelengths in (polar) methanol and (apolar) toluene are 498 and $417 \mathrm{~nm}$, respectively; the fluorescence band shift in response to solvent change from toluene to methanol is $3,900 \mathrm{~cm}^{-1}$.

Construction of T7 phage display library, as well as a T7 phage displaying a model peptide (-GSRVSCGGRDRPGCLSV), was as reported previously. ${ }^{2}$

\section{Construction of a fluorogenic library via the $10 \mathrm{BASE}_{\mathrm{d}}-\mathrm{T}$}

Gp10 based-thioetherification (10BASE $\left.\mathrm{d}_{\mathrm{d}} \mathrm{T}\right)$ on $\mathrm{T} 7$ phage was performed as described previously ${ }^{2}$ with minor modifications. Standard reaction conditions are the following: T7 phage particles (approximately $1.0 \times 10^{11}$ plaque-forming units) were well suspended by sonication or vortex in $0.25 \mathrm{~mL}$ of phosphate buffered saline (PBS) supplemented with final $0.26 \mathrm{M} \mathrm{NaCl}$. After centrifugation at 12,000 rpm for 5 minutes at room temperature, the supernatant was mixed with neutralized tris(2-carboxyethyl)phosphine (TCEP) aqueous solution at a final concentration of 0.63 $\mathrm{mM}$ at $4{ }^{\circ} \mathrm{C}$. For the optimization experiment in Fig. S1, Badan in dimethyl sulfoxide (DMSO) was added at various concentrations and the mixture was incubated at $4{ }^{\circ} \mathrm{C}$ for 3 hours in the dark. For the library construction, Badan was added to a final concentration of $0.58 \mathrm{mM}$. To inactivate the unreacted Badan, 2-mercaptoethanol was 
added to the mixture to a final concentration of $3.5 \mathrm{mM}$, and further incubated at $4{ }^{\circ} \mathrm{C}$ for 1 hour. The T7 phage particles were precipitated with a mixture of polyethylene glycol 6000 and sodium chloride to final concentrations of $5 \% \mathrm{w} / \mathrm{v}$ and $0.6 \mathrm{M}$, respectively. After centrifugation at $15,000 \mathrm{rpm}$ for 15 minutes at $4{ }^{\circ} \mathrm{C}$, the precipitate was dissolved in an appropriate buffer.

\section{Biotinylation of GST}

$15 \mathrm{~g} / \mathrm{L} \mathrm{GST}^{2}$ in PBS was mixed with Biotinamidohexanoic acid 3-sulfo-N-hydroxysuccinimide ester sodium salt (Sigma, MO, USA) at a final concentration of $1.5 \mathrm{mM}$ and left $4{ }^{\circ} \mathrm{C}$ overnight. For desalination, Zeba ${ }^{\mathrm{TM}}$ Spin Desalting Columns (Thermo Fisher Scientific, MA, USA) was used with centrifugation at 3,000 rpm for 4 minutes at $4{ }^{\circ} \mathrm{C}$. The biotinylation of GST was confirmed by Western blotting. From densitometric quantification reported previously, ${ }^{2}$ it was estimated that approximately 0.5 molecules of biotin were conjugated to a single GST molecule (data not shown).

\section{Biopanning}

Biopanning against GST was performed as described previously. $^{2}$ In brief, approximately $1.0 \times 10^{11}$ pfu of T7Select10 library (-S-G-G-G-X $3-C-X_{5-7}-C-X_{3} ; \mathrm{X}$ represents any randomized amino acid) was modified via the $10 \mathrm{BASE} \mathrm{d}_{\mathrm{d}} \mathrm{T}$. After modification, the T7 phage library was dissolved in selection buffer (PBS supplemented with $0.1 \% \mathrm{v} / \mathrm{v}$ TritonX-100). To remove non-specific binders (i.e. beads, streptavidin, and BSA binders), the modified T7 phage library was pre-incubated with streptavidin-coupled beads for 16 hours at $4{ }^{\circ} \mathrm{C}$, and then the supernatant was further incubated with the GST-immobilized beads for 15 hours. The latter beads were washed three times for 15 minutes in total with $0.2 \mathrm{~mL}$ of the selection buffer. The entire binding and washing processes were performed using an automated machine (Target Angler 8, Tamagawa Seiki, Japan). GST-bound phage was directly infected and amplified with E. coli BLT5403 strain. Increasing stringent conditions such as shortening the binding time (e.g. 2 hours at the final round) and increasing the washing 
frequency / time (up to ten times / 150 minutes in total at the final round), were applied stepwise to each round. After 5 rounds of biopanning, randomly chosen T7 phage monoclones were subjected to ELISA followed by DNA sequencing.

\section{Enzyme-linked immunosorbent assay (ELISA)}

ELISA was performed as described previously with minor modifications. ${ }^{2}$ Each well of 96-well plates (Maxisorp, Clear Flat-Bottom Immuno Nonsterile 96-Well Plates, Thermo Scientific) was coated with $5 \mu \mathrm{M}$ streptavidin in PBS at $4{ }^{\circ} \mathrm{C}$ overnight. After discarding the solution in each well, TBS supplemented with $0.05 \% \mathrm{v} / \mathrm{v}$ Tween-20 and $0.5 \% \mathrm{w} / \mathrm{v}$ BSA was applied to the well and incubated for 1 hour at $25^{\circ} \mathrm{C}$ with shaking using a maximizer (MBR-022UP, TAITEC, Japan). After washing with PBS supplemented with TritonX-100, biotinylated-GST $(0.15 \mathrm{nmol})$ was immobilized on each well. Approximately $1.0 \times 10^{11}$ pfu of T7 phage in TBS supplemented with $0.5 \%$ $\mathrm{v} / \mathrm{v}$ TritonX-100 was applied to the well and incubated for 1 hour at $25{ }^{\circ} \mathrm{C}$ with shaking using the maximizer. The plate was washed three times with TBS supplemented with $0.5 \% \mathrm{v} / \mathrm{v}$ TritonX-100, and then GST-bound phage was incubated with T7 tail fiber monoclonal antibody (1:4,000 dilution, Merck Millipore, Germany) and anti-mouse IgG horseradish peroxidase-linked antibody (1:4,000 dilution, Cell Signaling Technology, MA, USA). After washing with TBS supplemented with $0.5 \% \mathrm{v} / \mathrm{v}$ TritonX-100, substrate solution (0.05 M citric acid, $0.02 \mathrm{M}$ hydrogen peroxide, $0.4 \mathrm{mM}$ 2,2'-azino-bis(3-ethylbenzothiazoline-6-sulfonic acid) diammonium salt, $\mathrm{pH}$ 4) was added, and the absorbance was quantified using a microplate reader equipped with a $405 \mathrm{~nm}$ band-pass filter (Bio-Rad, CA, USA).

Among 16 randomly chosen single $\mathrm{T} 7$ phage plaques after the 5 rounds of biopanning, 15 clones had positive signals for GST binding only when Prodan was conjugated to the designated Cys on the displaying peptide (see the body text and Fig. 2). The remaining single clone (GTVQC GST, which possessed a weak amino-acid homology (underlined) with the best Prodan evolver 1 (i.e. NTVSC ${ }^{*}$ HGF) (Fig. S10). This means that some or most of the unidentical amino acids in $\underline{\mathbf{1}}$ (bold) are also essential for the specific binding to GST. 


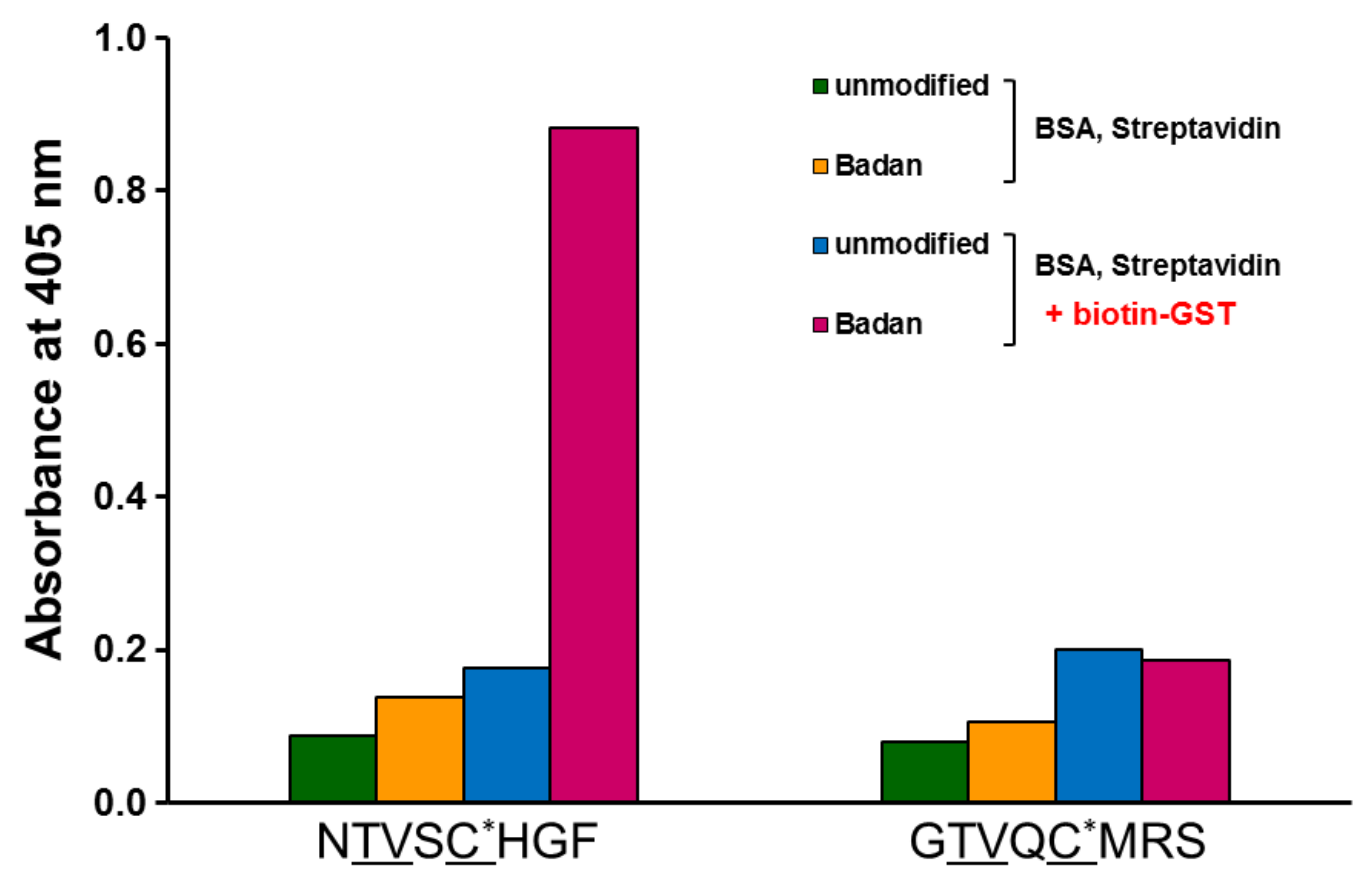

Figure S10. GST binding ability of the best and worst Prodan evolvers on T7 phage monoclone. The amino acid sequences of them are shown in the bottom, and their homology is underlined. These monoclones after 5 round of biopanning were reacted with Badan. Both the modified and unmodified monoclones were subjected to ELISA. Mixture of streptavidin and bovine serum albumin (BSA) served as mock proteins.

\section{Synthesis of Prodan-evolvers ( $\underline{1}-\underline{3})$}

Peptides ( $\mathrm{H}_{2} \mathrm{~N}-\mathrm{NTVSCHGF-OH}, \mathrm{H}_{2} \mathrm{~N}-\mathrm{NNPCTGF-OH}$, and $\left.\mathrm{H}_{2} \mathrm{~N}-\mathrm{PGPCG}-\mathrm{OH}\right)$ as synthons for $\underline{\mathbf{1}}, \underline{\mathbf{2}}$, and $\underline{\mathbf{3}}$, respectively, was synthesized and characterized by GenScript Inc (NJ, USA). Purity of the peptide was estimated to be above $90 \%$. For the reaction with Badan, each peptide was dissolved in phosphate buffer (30 mM phosphate, $\mathrm{pH} 7.4$ ) at a final concentration of $12 \mathrm{mM}$ in the presence of $10 \mathrm{mM}$ Badan and neutralized 1 $\mathrm{mM}$ TCEP. The mixture was reacted for 6 hours at room temperature in the dark with vigorous shaking, acidified with aqueous formic acid, and purified with reverse-phase high performance liquid chromatography (LC-20AD, Shimadzu, Japan) equipped with a 
XTerra Prep MS C18 column $(10 \times 50 \mathrm{~mm}$, Waters $)$. Each conjugate was separated using a $0-100 \%$ linear gradient of methanol containing $0.1 \%$ trifluoroacetic acid during $12 \mathrm{~min}$ at a flow rate of $4 \mathrm{ml}$ per minute. It was lyophilized completely and characterized by LC-MS/MS (liquid chromatography-mass spectrometry/mass spectrometry) (see below; Fig. S11). Purity was estimated to be above 90\%. The overall yields of $\underline{\mathbf{1}}, \underline{\mathbf{2}}$, and $\underline{\mathbf{3}}$, were 32,42 , and $60 \%$, respectively. After the purification, the UV-visible absorption spectra of the Prodan-evolvers were measured. An example is shown in Fig. S12.

(A) NTVSC ${ }^{*} H G F(1)$

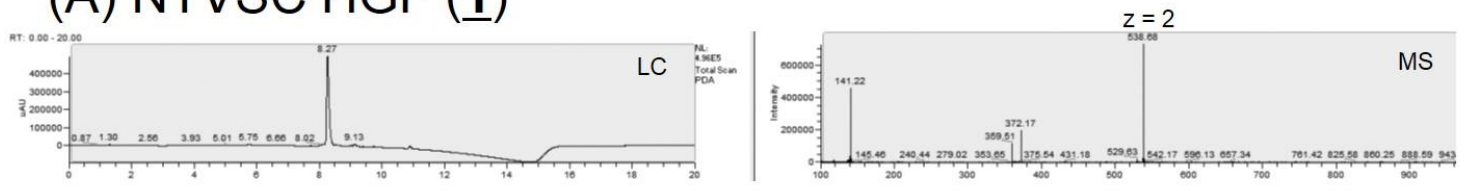

(B) NNPC*TGF (2)
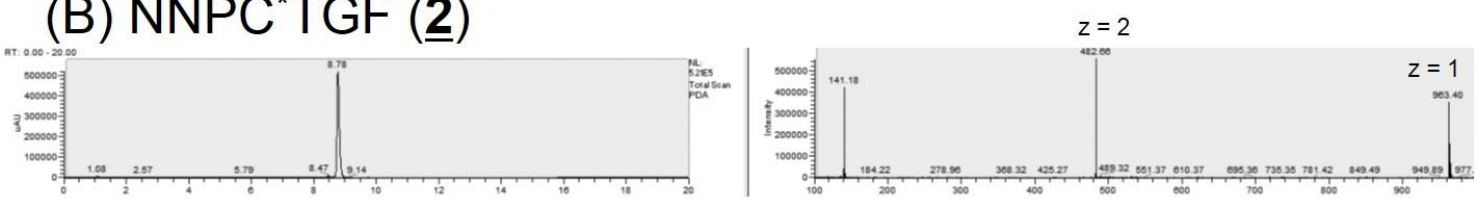

(C) PGPC ${ }^{*} G(\underline{3})$

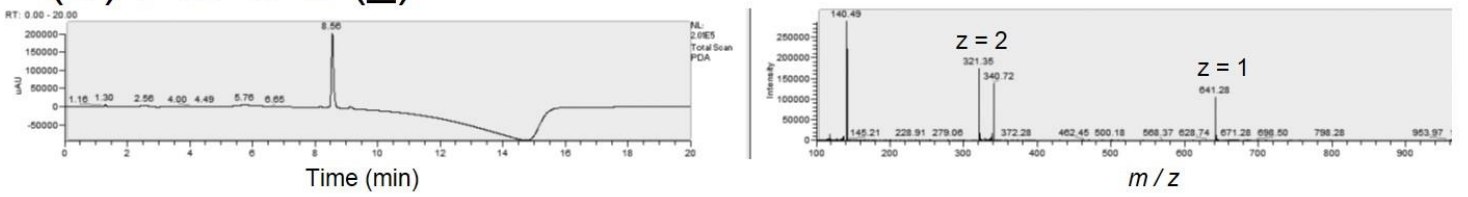

Figure S11. LC-MS spectra of purified Prodan-evolvers $\underline{\mathbf{1}}-\underline{\mathbf{3}}$. Identification by MS/MS fragmentation analysis was also carried out (data not shown).

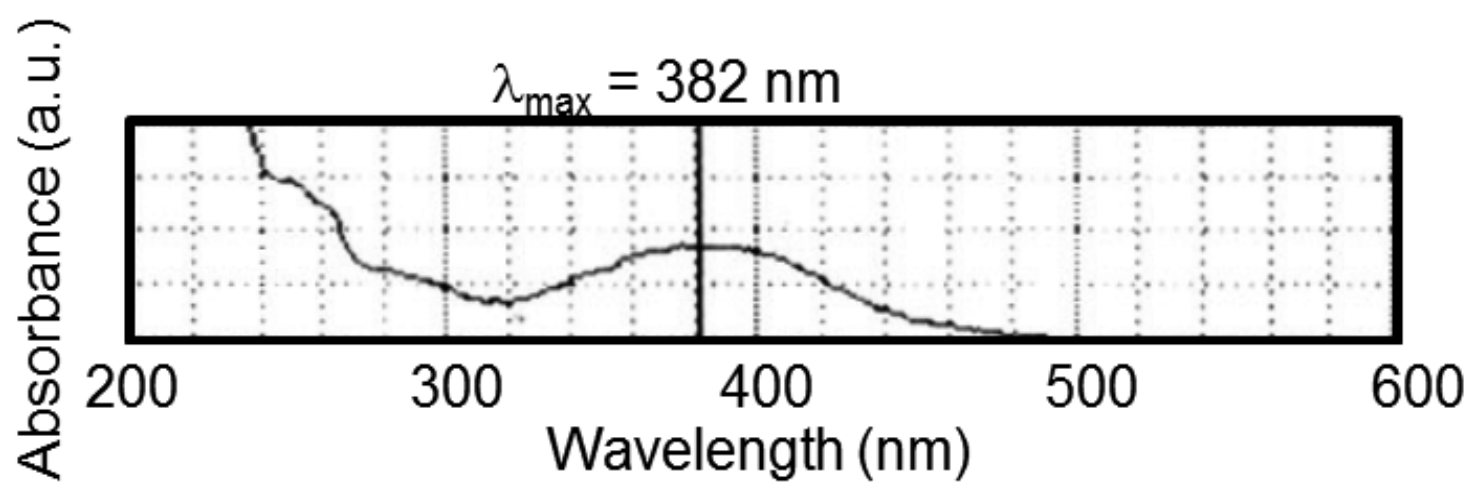

Figure S12. UV-visible absorption spectrum of the Prodan-evolver $\underline{\mathbf{1}}$. The maximum 
absorption peak was at $382 \mathrm{~nm}$ with molar absorption coefficient of $1.5 \times 10^{4} \mathrm{~mol}^{-1} \mathrm{~L}$ $\mathrm{cm}^{-1}$, and the peak was broadened to visible region. The coefficient at a typical blue-laser excitable wavelength, $405 \mathrm{~nm}$, was $1.3 \times 10^{4} \mathrm{~mol}^{-1} \mathrm{~L} \mathrm{~cm}^{-1}$.

For the Prodan-evolver (1), circular dichroism (CD) spectra were also recorded before and after the conjugation with Badan, as reported previously. ${ }^{3}$ In either of them, no conspicuous peaks representative of tertiary structures were observed (data not shown).

\section{Isothermal titration calorimetry (ITC)}

ITC experiment was performed using MicroCal iTC $_{200}$ (GE Healthcare, CT, USA). ${ }^{3}$ GST was enriched using an ultrafiltration column (Vivaspin column $20 \mathrm{MWCO} 10 \mathrm{kDa}$, GE Healthcare), and the buffer was changed to phosphate buffer (20 mM phosphate, $\mathrm{pH}$ 7.4, $50 \mathrm{mM} \mathrm{NaCl}, 1 \mathrm{mM}$ TCEP). Protein concentration was determined by Bradford assay. For titration experiment, proteins and ligands were diluted into $25 \mu \mathrm{M}$ and 250-600 $\mu \mathrm{M}$, respectively. Titrations were performed at $30^{\circ} \mathrm{C}$. Injection parameters were the following: $2 \mu \mathrm{L}$ volume, $4 \mathrm{sec}$ duration, $60 \mathrm{sec}$ spacing, and $5 \mathrm{sec}$ filter period. Reference power was set to $5 \mu \mathrm{cal} / \mathrm{s}$. Data were analyzed on Origin software 7.0 (MicroCal). Curve fitting was performed using 1:1 interaction model.

\section{Fluorescence titration assay}

All fluorescence measurements were performed on a NanoDrop 3300 (Thermo Scientific) at room temperature. For measurement of fluorescence intensity change, various concentrations of GST were added to each Prodan-evolver $(5 \mu \mathrm{M})$ in PBS. The fluorescence spectra between 400 and $600 \mathrm{~nm}$ were measured by excitation at $365 \pm 10$ $\mathrm{nm}$. Relative fluorescence intensity changes at $551 \mathrm{~nm}$ were calculated by an equation of $\left(F_{\mathrm{x}}-F_{0}\right) / F_{0}$, where $F_{\mathrm{x}}$ and $F_{0}$ are fluorescence intensity of each Prodan-evolver with and without protein (i.e., GST, BSA, streptavidin, acylase, and casein), and were analyzed on GraphPad Prism 6 (GraphPad Software, Inc., CA, USA) to obtain dissociation constant $\left(K_{\mathrm{D}}\right)$. All of the Prodan-evolvers $(\underline{\mathbf{1}}-\underline{\mathbf{3}})$ showed remarkable color 
change and worked as indicators upon GST binding (Fig. 2A and S4A), whereas TMR-evolvers $^{2}$ did not (data not shown). To confirm the GST specificity of the best Prodan evolver $\underline{\mathbf{1}}$ thoroughly, the above fluorescence titration measurements were also performed with several target-unrelated proteins (i.e., lipase, chicken egg avidin, carbonic anhydrase, human serum albumin, and ribonuclease A) other than those shown in Fig. 3B, resulting that none of them showed remarkable color-changing and turning-on (data not shown).

\section{Nucleic magnetic resonance (NMR) measurements}

NMR experiments including ${ }^{1} \mathrm{H}-{ }^{1} \mathrm{H}$ COSY and STD measurements were performed at $30{ }^{\circ} \mathrm{C}$ by using a $500 \mathrm{MHz}$ spectrometer (JNM-ECA500, Jeol Resonance, Japan). Proton assignments of Prodan-evolver (1) were performed by using a conventional set of 2D spectra: 2D ${ }^{1} \mathrm{H}-{ }^{1} \mathrm{H}$ COSY, totally correlated spectroscopy (TOCSY) with $50 \mathrm{~ms}$ mixing time, nuclear Overhauser effect correlated spectroscopy (NOESY) with $0.5 \mathrm{~s}$ mixing time. Prodan-evolver $(\underline{\mathbf{1}} ; 0.60 \mathrm{mM})$ was dissolved in $10 \mathrm{mM}$ phosphate $(\mathrm{pH} 7.4)$ $/ \mathrm{D}_{2} \mathrm{O}$ with $7 \%$ DMSO- $d_{6}$. For the STD measurements, selective saturations of GST with various saturation time were achieved by a train of Gauss-shaped pulses of $60 \mathrm{~ms}$. On-resonance and off-resonance irradiations of GST were performed at a chemical shift of 0.15 and $-10 \mathrm{ppm}$, respectively. The STD spectra were taken by subtraction of the on- and off-resonance spectrum. The total scan number of each STD measurement was ca. 7000, and domain data points were 4096. For the COSY measurement, a molar concentration of $0.14 \mathrm{mM}$ was employed for $\underline{\mathbf{1}}$, GST, or both of them in the same buffer. The spectra were analyzed on Jeol Delta-5 program.

\section{Molecular docking simulations of the Prodan-evolver (1) to GST using Autodock Vina:}

Docking of NTVSC*TGF to glutathione-S-transferase (PDB: 1UA5) was performed with Autodock Vina as follows. The Prodan-evolver (1) was created in Sybyl X2.1.1 (Certara Inc, Princeton, NY, USA) and converted to a pdbqt file format using Auto 
DockTools (v1.5.6, http:mgltools.scripps.edu). The target GST protein was stripped of the co-crystalized $\mathrm{GSH}, \mathrm{SO}_{4}$, and water molecules and converted to a pdbqt file format using Auto DockTools. The ligand and protein input files were entered and docked using Autodock Vina (http://vina.scripps.edu/index.html) with target box parameters of size 22, 20, 20 A centered at 20,80,6. Nine separate poses resulted in docking with free energy in the range -7.1 to $-6.8 \mathrm{kcal} / \mathrm{mol}$. The image shown in Fig. S10 is the pose with the lowest docking energy with the ligand shown as a stick figure and the target shown as a cartoon with extruding side chains (Pymol v1.7.6, http://www.schrodinger.com/pymol/).

\section{Supplemental references}

(1) Klymchenko, A. S.; Mely, Y. Prog. Mol. Biol. Transl. Sci. 2013, 113, $35-58$.

(2) Fukunaga, K.; Hatanaka, T.; Ito, Y.; Taki, M. Mol. BioSyst. 2013, 9, 2988-2991.

(3) Fukunaga, K.; Hatanaka, T.; Ito, Y.; Minami, M.; Taki, M. Chem. Commun. 2014, 50, 3921-3923. 